\title{
Apparition d'encéphalomyélite aviaire au Sénégal
}

\author{
E. Cardinale ${ }^{1}$ J.F. Dayon ${ }^{2}$ Y. Kaboret ${ }^{3}$ \\ G. Pene ${ }^{4}$ M. Faye ${ }^{5}$ B. Doyen ${ }^{6}$
}

Mots-clés

Volaille - Poulette - Encéphalite Vaccination - Animal reproducteur Syndrome de chute de ponte - Sénégal.

\begin{abstract}
Résumé
Des cas d'encéphalomyélite aviaire ont été mis en évidence sur des poussins, poulettes futures pondeuses, en novembre 1997. C'était la première fois que cette maladie était diagnostiquée au Sénégal. Les lésions classiques de cette virose révélées lors du diagnostic histologique ont confirmé sa présence. La vaccination contre l'encéphalomyélite n'était pas obligatoire et certains couvoirs ne vaccinaient pas leurs reproducteurs. L'existence de cette pathologie semble imposer dorénavant une vaccination de tous les reproducteurs du Sénégal. Parallèlement, se pose aussi le problème de la vaccination des poulettes, futures pondeuses. En effet, le syndrome de chute de ponte sévit à I'heure actuelle sur le territoire et le laboratoire doit, en premier lieu, effectuer un diagnostic différentiel avec la maladie de N ewcastle et la bronchite infectieuse avant de proposer des recommandations précises. La technique Elisa doit être utilisée pour cela.
\end{abstract}

\section{INTRO DUCTION}

L'encéphalomyélite aviaire est une maladie virale des poulets, des dindons, des faisans et des cailles. Elle se caractérise chez les jeunes oiseaux par une ataxie progressive évoluant vers une paralysie et, classiquement, par des tremblements de la tête et du cou. Les adultes infectés ne présentent, en général, aucun signe. Cette maladie est répandue de façon cosmopolite mais est actuellement maîtrisée par des vaccins efficaces. Elle est provoquée par un picornavirus qui peut se transmettre horizontalement entre les poussins par l'intermédiaire de litière contaminée ou par le personnel, ou verticalement par les œufs (8). A la connaissance des auteurs, cette pathologie n'a jamais été mise en évidence au Sénégal. Cette réalité s'explique par le fait que les poussins utilisés jusqu'à ce jour étaient importés, issus de reproducteurs exotiques vaccinés contre l'encéphalomyélite aviaire.

1. Cirad-emvt / Isra-Lnerv, BP 2057, Dakar-Hann, Sénégal

2. Projet Prodec, Mission française de coopération, Dakar, Sénégal

3. Eismv, BP 5077, Dakar, Sénégal

4. BP 18221, Pikine, Sénégal

5. Projet Plan international, BP A152, Thiès, Sénégal

6. BP 18043, Pikine, Sénégal

\section{LES FAITS}

Les premiers signes de la maladie sont apparus le 15 novembre 1997 dans six élevages $*$ du pourtour Dakarois (zone des Niayes) et à Thiès, soit dans un rayon de moins de $100 \mathrm{~km}$ autour de Dakar. Les manifestations ont concerné des poussins (poulettes, futures pondeuses) âgés de 21 à 25 jours (tableau I). Ces animaux étaient issus du même couvoir, de deux lots de reproducteurs de souches différentes mais d'âge similaire qui ont manifesté des chutes de ponte.

\section{LES SYM PTO M ES O BSERVES}

Les poussins ont présenté une incoordination motrice. Ils s'asseyaient souvent sur leurs articulations tibio-métatarsiennes (figure 1) ou adoptaient une position en décubitus latéral, la tête étant maintenue perpendiculairement au sol (figure 2). Certains poussins ne se déplaçaient pas, atteints de paralysie flasque. D'autres émettaient des cris plaintifs.

De légers tremblements, particulièrement bien mis en évidence lorsque l'animal se trouvait dans la paume de la main, agitaient la tête et le cou.

* Les informations présentées ne concernent que les élevages qui ont été visités par l'équipe du laboratoire qui a été directement contactée par les éleveurs. Mais il semblerait que plusieurs autres exploitations aient été touchées 


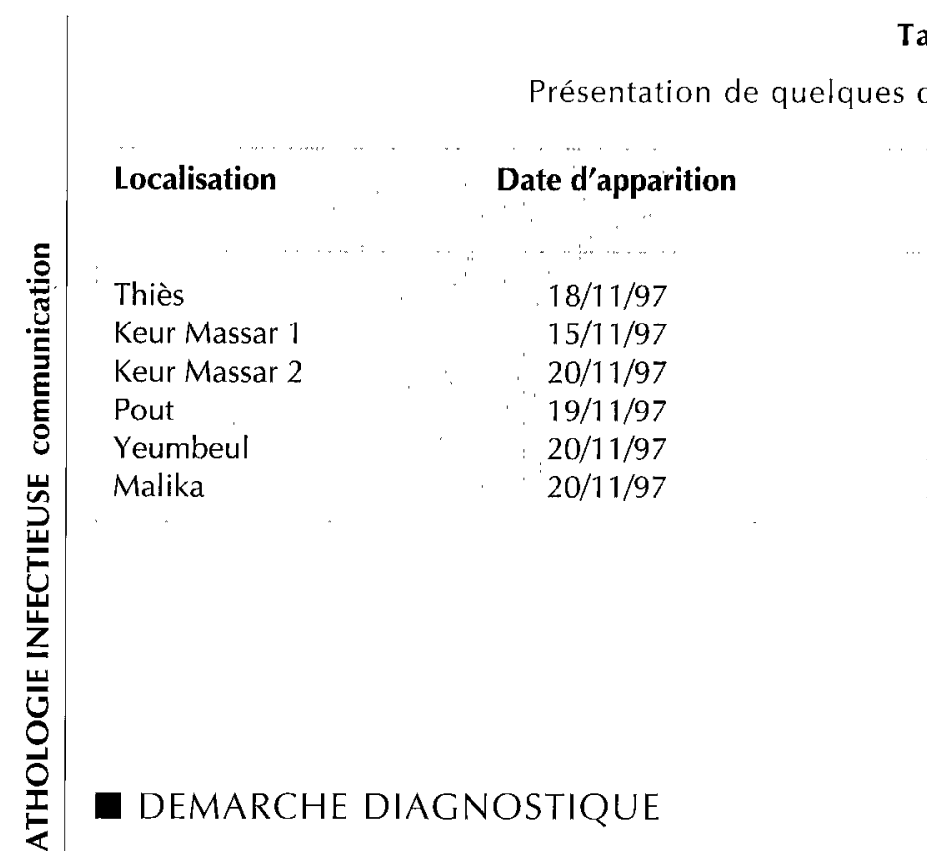

\section{Examen nécropsique}

Les autopsies ont porté sur 60 sujets (10 par élevage) sur lesquels aucune lésion caractéristique notable n'a pu être détectée.

\section{Examen histologique}

Des prélèvements du pancréas, du proventricule, du gésier et du cerveau ont été réalisés sur les sujets autopsiés et ont été fixés au formol à 10 p. 100.

Deux lots de prélèvements (Thiès et Keur Massar 1) ont été envoyés au service d'anatomopathologie du laboratoire de développement et d'analyse ( $\mathrm{Lda} 22$ ) de Ploufragan et les quatre autres lots au service d'anatomopathologie de l'Eismv* de Dakar.

Le descriptif lésionnel du lot de Thiès au niveau des encéphales a rapporté l'existence de petits foyers de neuronophagie, des manchons lymphoïdes périvasculaires nets et bien constitués au niveau du bulbe, des infiltrats inflammatoires non significatifs de la muqueuse des proventricules et des gésiers et de rares éléments inflammatoires au niveau des pancréas. Ces lésions microscopiques étaient suffisamment significatives pour confirmer une encéphalomyélite aviaire.

Les lésions mises en évidence dans les organes du deuxième lot étaient les suivantes : petits manchons lymphoïdes périvasculaires avec légère gliose sur les encéphales, petits infiltrats lymphoïdes pléomorphes dans la musculeuse des gésiers ainsi que dans le pancréas. Ces résultats indiquaient la présence d'une infection virale trop discrète ou trop débutante pour confirmer, par la seule histologie, l'existence d'une encéphalomyélite.

En revanche, les quatre lots d'organes envoyés à l'Eismv et prélevés sur des poussins un peu plus âgés (30 jours) ont présenté les lésions microscopiques caractéristiques de la maladie : une infiltration musculaire multifocale dans la musculeuse du gésier (figure 3) et dans l'interstitium pancréatique, une gliose diffuse et des manchons lymphoïdes périvasculaires dans le cerveau (figure 4), une chromatolyse des neurones dans le pont cérébral et une perte neuronale dans le cervelet (figure 5).

* Ecole inter-états des sciences et de médecine vétérinaire
Tableau I

\begin{tabular}{|c|c|c|c|c|}
\hline \multirow[t]{2}{*}{ Age } & \multicolumn{2}{|c|}{ Mortalité } & \multicolumn{2}{|c|}{ Morbidité } \\
\hline & $\%$ & (n) & $\%$ & (n) \\
\hline 25 jours & 12 & $(120)$ & 25 & $(250)$ \\
\hline 21 jours & 17 & (255) & 37 & (555) \\
\hline 21 jours & 7 & (105) & 12 & (180) \\
\hline 23 jours & 10 & $(120)$ & 15 & (180) \\
\hline 25 jours & 25 & $(350)$ & 50 & $(700)$ \\
\hline 25 jours & 45 & $(630)$ & 50 & $(700)$ \\
\hline
\end{tabular}

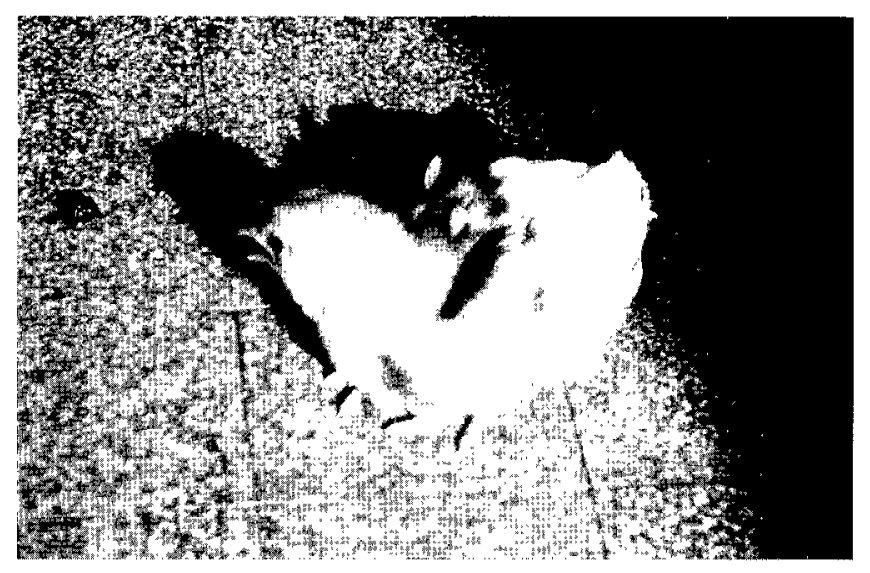

Figure 1 : position caractéristique du poussin assis sur ses tibio-métatarses.

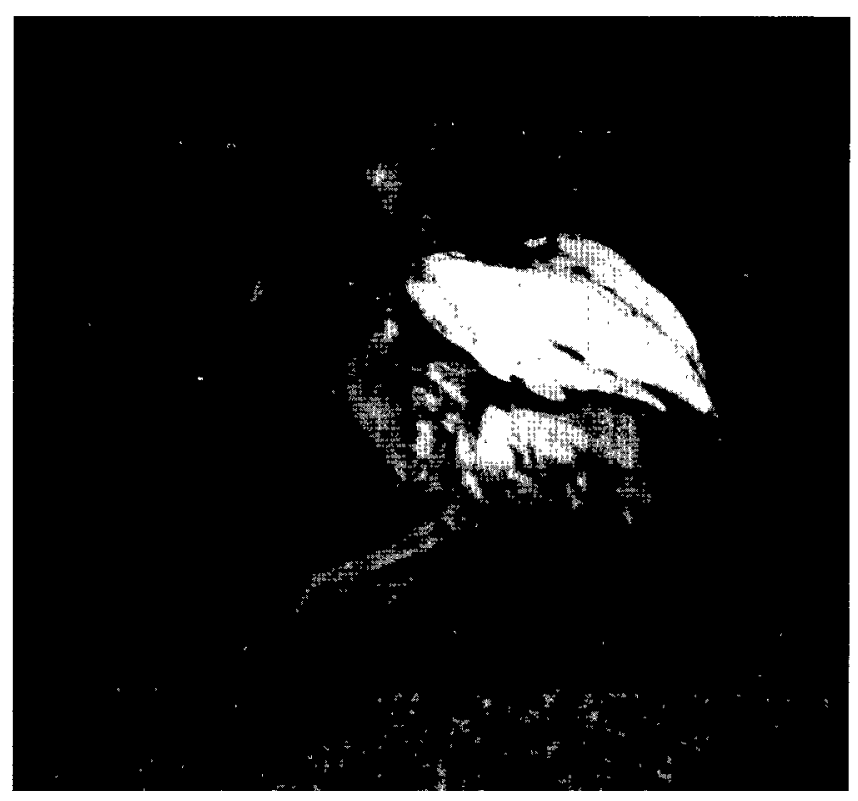

Figure 2 : position en décubitus latéral avec la tête maintenue perpendiculairement au sol. 


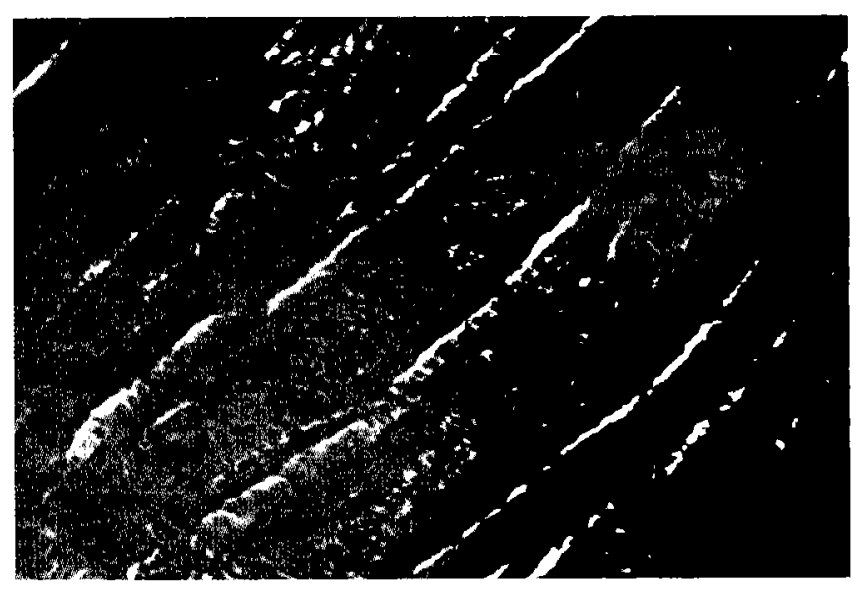

Figure 3 : infiltration lymphoïde multifocale dans la musculeuse du gésier.

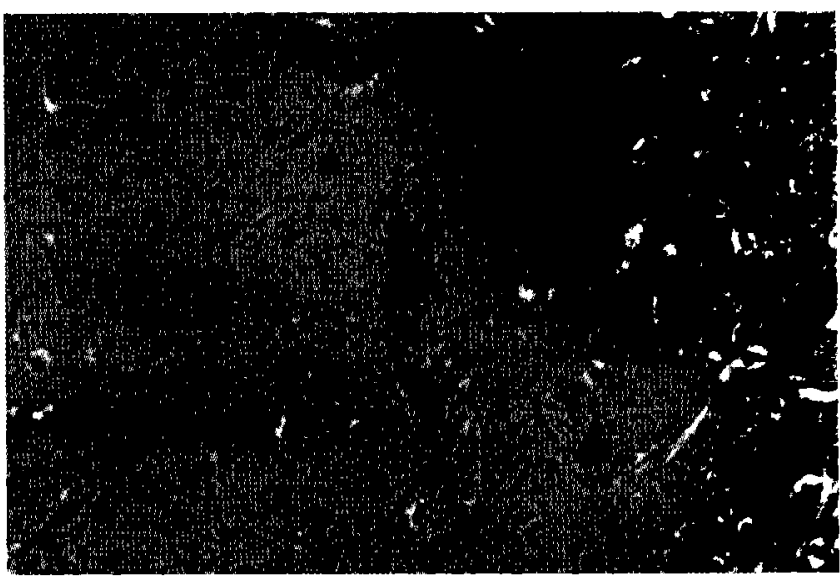

Figure 4 : manchons lymphö̈des périvasculaires dans le cerveau.

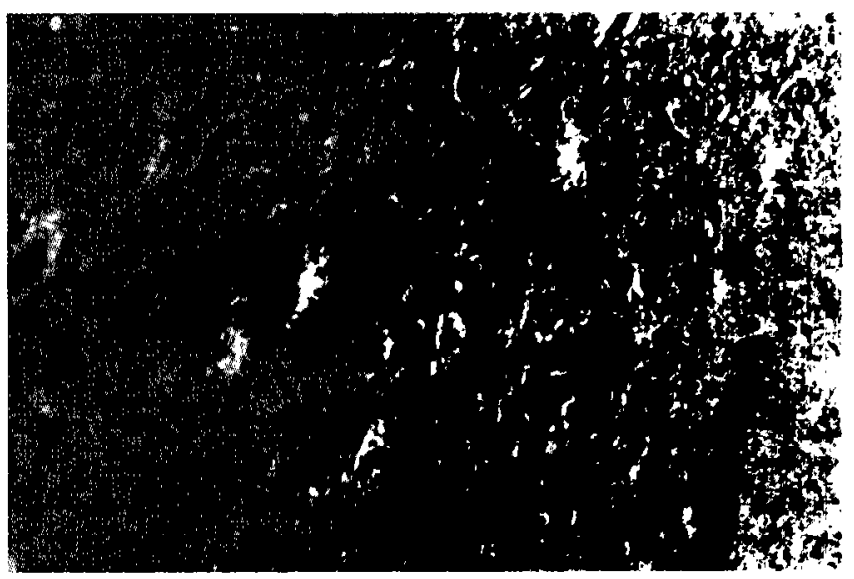

Figure 5 : chromatolyse des neurones.

\section{DISCUSSION}

L'apparition brutale de cette pathologie au Sénégal a révélé la présence du virus sur le territoire, présence jusqu'alors masquée par la protection efficace des poussins en grande majorité importés soit sous forme d'œufs à couver, soit en poussin d'un jour.

Ces importations provenaient presque toutes de France ou de Belgique, pays où des programmes de contrôle des reproducteurs ont été mis en place et où certaines vaccinations ont été rendues obligatoires.
Au Sénégal, depuis la dévaluation du franc CFA qui a renchéri le coût des importations, la tendance a été d'élever des reproducteurs sur le territoire et la part de marché des poussins produits localement s'est étendue de plus en plus. A ce jour, le contrôle officiel des couvoirs et des reproducteurs n'a pas été mis en place.

En ce qui concerne l'encéphalomyélite aviaire, aucune recommandation n'a été faite de la part des services vétérinaires. Certains couvoirs ont assuré la vaccination de leurs reproducteurs, d'autres ne l'ont pas pratiquée. L'apparition de cette maladie a été rendue possible par la mise en place sur le terrain d'un grand nombre de poussins non protégés. La vaccination 'des reproducteurs n'ayant pas été obligatoire, cette insuffisance de protection était due soit à l'absence de vaccination soit à une mauvaise vaccination. L'existence de cette maladie impose désormais l'application d'un protocole vaccinal.

La vaccination des poulettes futures pondeuses ou reproductrices fait appel à un vaccin vivant à administrer pendant la phase d'élevage, entre dix et seize semaines d'âge (4). En effet, la protection conféré par des reproducteurs vaccinés durant la ponte protègent les descendants pendant le jeune âge (environ deux mois), la vaccination pendant cette période n'est donc pas recommandée. Cependant, une vaccination trop tardive (moins de quatre semaines avant le début de la ponte) risque d'entraîner des problèmes de chute de ponte en raison de la persistance du virus vaccinal dans le troupeau encore insuffisamment protégé.

Le vaccin peut être administré par voie orale dans de l'eau de boisson, en respectant les recommandations générales de cette méthode : s'assurer de l'absence de désinfectant et d'ions métalliques en excès dans l'eau, assoiffer au préalable les animaux, utiliser des abreuvoirs propres et en nombre suffisant, ajouter de la poudre de lait... (3). Des travaux récents ont déjà montré que cette technique n'était pas forcément maîtrisée par les éleveurs ou les employés d'élevages (1). Pour les reproductrices, le succès de cette vaccination est capital pour l'avenir de la progéniture. Elle doit être impérativement réalisée sous le contrôle du technicien ou du vétérinaire responsable afin d'en assurer sa bonne réalisation. Certains laboratoires proposent également d'avoir recours à une vaccination par des méthodes individuelles : instillation par voie buccale pour l'encéphalomyélite vaccin Nobilis, goutte dans l'œil pour le vaccin encéphalomyélite TAD. En plus des avantages procurés par les mćthodes individuclles (meilleure homogénéité, absence de problème pour la dilution du vaccin), dans. le cas de l'encéphalomyélite, il est possible de ne vacciner qu'une partie du troupeau, le reste des animaux se vaccinant par contact (5). Par exemple, avec le vaccin Nobilis, il est recommandé, sur 500 sujets, d'en vacciner 20 qui sont ensuite répartis de façon homogène dans le troupeau. Dans le cas de petits effectifs et lorsque l'on maîtrise mal la vaccination par l'eau de boisson, cette méthode est certainement préférable. L'efficacité de la vaccination peut néanmoins être contrôlée au laboratoire.

Il faut aussi noter que des vaccins inactivés ont été développés pour protéger des vise'dux déjà en ponte (2). L'utilisation du vaccin vivant reste toutefois la méthode la plus utilisée.

Parallèlement aux reproducteurs, se pose le problème de la vaccination des poulettes futures pondeuses. Les descendants des reproducteurs ne sont protégés que pendant les huit premières semaines et les pondeuses issues de ces poulettes peuvent, par conséquent, manifester des chutes de ponte (seul signe clinique de la maladie manifesté chez les adultes). Il est donc recommandé de vacciner ces animaux puisque le virus est présent sur le territoire. Cependant, au niveau du laboratoire, la responsabilité du picornavirus dans des pathologies liées aux chutes de ponte n'a pas pu, pour le 
moment, être mise en évidence en raison de l'absence de symptômes cliniques caractéristiques chez les adultes, du faible nombre de prélèvements parvenus et de l'ignorance de l'existence de cette maladie. Il importe donc de mieux connaître les facteurs responsables des chutes de ponte chez les pondeuses au Sénégal. En effet, pendant la période de ponte, le diagnostic différentiel avec la maladie de Newcastle et la bronchite infectieuse principalement doit être effectué. Pour cela, le laboratoire a besoin de mener un suivi sérologique permettant de détecter la présence de ces différents virus dans les élevages et de déterminer leur importance respective dans le syndrome de chute de ponte. Cette détection peut par la suite être confirmée par des examens complémentaires. En parvenant à quantifier économiquement les chutes de ponte liées à l'encéphalomyélite aviaire, il sera plus aisé de recommander l'administration d'un vaccin à tous les élevages de poulettes.

\section{CONCLUSION}

$\mathrm{Au}$ vu de ces informations, le laboratoire de pathologie aviaire de Dakar doit s'équiper rapidement pour répondre aux questions précédentes. Le choix devrait porter sur la méthode Elisa, considérée comme une méthode de référence (6) et dont la technique est mâ̂trisée par le personnel de laboratoire. Cette méthode permettra de vérifier l'efficacité du vaccin par le contrôle des titres en anticorps post-vaccinaux et de déceler le passage d'un virus sauvage par la présence d'anticorps sur des animaux non vaccinés ou par une augmentation anormale des titres en anticorps sur des prélèvements séquentiels (7).
BIBLIO GRAPHIE

1. ARBELOT B., DAYON J.F., MEROUANI N., KABORE Y., 1997. Etude des programmes vaccinaux réalisés en aviculture au Sénégal. In :

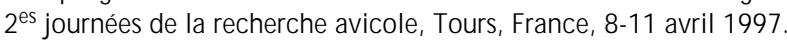

2. BUTTERFIELD W.K., LUGINBUHL R.E., HEMBOLDT C.F., SUMNER F.W., 1961. Studies on avian encephalomyelitis. III. Immunization with an inactivated virus. Avian Dis., 5: 445-450.

3. FOURNIER D., LEGROS F.X., VANMARCKE J., 1995. In : Rencontres internationales de production avicole, Nantes, France, 4 octobre 1995, p. 69-123.

4. JORDAN F.T.W., GOODERHAM K.R., MC FERRANET J.B., 1990. Diseases associated with the Picornaviridae. In: Jordan F.T.W. ed., Poultry diseases, 3rd Edition. London, UK, Baillière Tindall, p. 167-176.

5. MEISSONIER E., DEVISME P., JOIN-LAMBERT P., 1995. Encéphalomyélite vaccin Nobilis. In : Dictionnaire des médicaments vétérinaires. Maisons-Alfort, France, Editions du point vétérinaire, p. 274.

6. NICHOLAS R.A.J., REAM A.J., THORTON D.H., 1987. Detection of infectious avian encephalomyelitis virus in chick embryo neuroglial cell cultures. Arch. Virol., 96: 283-287.

7. SMART I.J., GRIX D.C., BARR D.A., 1986. The application of the ELISA to the diagnostic and control of avian encephalomyelitis. Aust. vet. J., 63: 297-299.

8. VENNE D., SILIM A., 1992. Encéphalomyélite aviaire. In : Manuel de pathologie aviaire. Maisons-Alfort, France, Ecole nationale vétérinaire, p. 139-141.

Reçu le 27.1.98, accepté le 24.3.99

\section{Summary}

Cardinale E., Dayon J.F., Kaboret Y., Pene G., Faye M., D oyen B. Appearance of avian encephalomyelitis in Senegal

Avian encephalomyelitis cases have been detected in chicks (future laying hens) in November 1997. It was the first time that the disease was diagnosed in Senegal. Typical lesions of the disease were revealed during the histological diagnosis and confirmed its presence. Vaccination against encephalomyelitis was not mandatory and some hatcheries did not vaccinate their breeding animals. The presence of the disease seems to require from now on a mandatory vaccination of all breeding animals in Senegal. In addition, there is the problem of vaccinating pullets, i.e. future laying hens. Actually, the egg drop syndrome is currently affecting the country, and the laboratory must first differentiate between encephalomyelitis, N ewcastle disease and infectious bronchitis before making precise recommendations. In this respect, the ELISA technique will have to be used.

Key words: Poultry - Hen - Encephalitis - Vaccination Breeding stock - Egg drop syndrome - Senegal.

\section{Resumen}

Cardinale E., Dayon J.F., Kaboret Y., Pene G., Faye M., Doyen B. Aparición de encefalomielitis aviar en Senegal

En noviembre 1997, se demostraron casos de encefalomielitis aviar en pollitos, pollas futuras ponedoras. Es la primera vez que se diagnostica esta enfermedad en Senegal. Las lesiones clásicas de esta virosis, observadas durante el diagnóstico histológico, confirmaron su presencia. La vacunación contra la encefalomielitis no era obligaría y ciertos establecimientos no vacunaban a los reproductores. La existencia de esta patología parece imponer una futura vacunación de todos los reproductores en Senegal. Paral elamente, se presenta también el problema de la vacunación de las pollas, futuras ponedoras. En efecto, actualmente se observa en campo un síndrome de caída de la postura y, antes de proponer recomendaciones precisas, el laboratorio debe, en primer lugar, efectuar un diagnóstico diferencial con la enfermedad de N ewcastle y la bronquitis infecciosa. Para responder a esta interrogante, se utilizará el método ELISA.

Palabras clave: Ave de corral - Gallina - Encefaliltis - Vacunación - Animal reproductor - Síndrome de caída de la postura Senegal. 